\title{
ARTICLE \\ Analysis of the Dilemma and Strategies of Elderly Patients Access to Outpatient Services - Based on the Examples from three Grade A Tertiary Hospitals in Jiangxi Province
}

\author{
Zheng Wang ${ }^{1,2^{*}}$ Mengyun Xu ${ }^{1,2}$ Zhiyan Zou ${ }^{1,2}$ \\ 1. The Jiangxi University of Finance and Economics, Nanchang, Jiangxi, 330013 China \\ 2. Monash University, Melbourne, Victoria, 3145, Australia
}

\section{ARTICLE INFO}

Article history

Received: 27 July 2020

Accepted: 30 July 2020

Published Online: 31 August 2020

Keywords:

Elderly patients

Outpatient

Dilemmas of accessing to medical services

\begin{abstract}
Objective: To identify the dilemma of elderly patients' access to outpatient services, develop strategies to improve the environment and functions of the outpatient department, and encourage the elderly to access medical services independently. Methods: By observing and interviewing, this paper studies the environment, behavior, and experiences of elderly patients when accessing medical services, identifies and classifies the key issues, and provides corresponding suggestions. Results: Existing signs and voice prompt systems fail to guide elderly patients to access to medical services; Elderly patients have difficulty in finding places to transit and rest when accessing to outpatient services; Elderly patients have problems in using AI (artificial intelligence) technologies when they access to outpatient services; There are communication barriers between elderly patients and medical staffs. Conclusion: Optimizing the guiding signs and voice prompt systems according to the characteristics of elderly patients; Designing the areas of transition and rest reasonably; Enhancing the ability of elderly patients to use self-service equipment; Promoting the medical treatment process to the elderly in a humanized way.
\end{abstract}

\section{Purpose and Significance}

$\mathrm{I}$ n 2016, the Communist Party of China Central Committee and the State Council released the 'Healthy China 2030' blueprint, which put forward the goals of Healthy China. In 2017, the $19^{\text {th }}$ National Congress of the Communist Party of China made a decision-making plan to implement the Healthy China Strategy. On the one hand, it emphasized the provision of high-quality and efficient medical services; on the other hand, it pointed out that it was necessary to strengthen the health services of critical populations and actively respond to the population aging. In 2019, the State Council issued a guideline to implement the Healthy China Strategy, which states that the government should maintain the whole life cycle health and promote the health conditions of the elderly. The medical care and health conditions of the elderly have received unprecedented attention. The critical issue of providing high-quality and efficient medical services for elderly patients needs to be solved urgently. With the growth of

\footnotetext{
*Corresponding Author:

Zheng Wang,

The Jiangxi University of Finance and Economics, Nanchang, Jiangxi, 330013 China;

Monash University, Melbourne, Victoria, 3145, Australia;

Email: 2562275375@qq.com
} 
the age, the elderly show increasing demands of medical services. Thus, creating a suitable environment for elderly patients to access medical services independently can become an essential part of the reform of the medical and health systems in the future. Compared with hospitalization and emergency treatment, elderly patients make more frequent use of outpatient services and are more likely to access medical treatment independently in the outpatient service department. Therefore, it is of great practical significance to analyze the difficulties of elderly patients accessing medical services from the outpatient department. It is also more convenient for the research team to observe and interview.

\section{Materials and Methods}

\subsection{Research Object and Method}

By the end of 2018, the number of elderly people over 65 years old in Nanchang was 593,400, accounting for 10.7\% of this city's total population. The proportion of the elderly population in Nanchang stays similar to the national level, and they both showed an upward trend year by year. The rate of economic development and the per capita income in Nanchang is in the mid-level among provincial capital cities in China. Thus, it is reasonable and representative to take the elderly in Nanchang as the research object.

From November 2018 to October 2019, the research team took advantage of weekly hospital social worker placements to analyze the outpatient environment of three Grade A tertiary hospitals in Nanchang through participatory observation and interview research methods. The research is not only beneficial to the horizontal comparison between medical institutions but also beneficial to the contrast between the current situation of outpatient medical service supply and the medical demands of the elderly.

The observation objects include the outpatient lobby partitions, the efficiency of guiding signs and voice prompt systems, the settings of spaces for transition and rest, the classification and quantity of service windows, the utilization of self-service equipment, the allocation of medical staff, and their attitudes. The interviewees include the elderly patients who access outpatient services and the medical staff who work for the outpatient department (including volunteers and hospital social workers). The interview focuses on the elderly's experience, habits, and difficulties when accessing outpatient services and the problems of medical staff in the outpatient department.

\subsection{Observed Indicator}

In China, hospitals are classified into three grades accord- ing to the resource allocation and service capacity. Primary and secondary hospitals can be further classified into Grade A, B and C, tertiary hospitals can be further classified into Grade S, A, B and C. Hospital F, Hospital R, and Hospital Z in Nanchang are all Grade A tertiary hospitals, with concentrated medical resources, a high volume of outpatients, and many elderly patients.

As one of the first Grade A tertiary referral hospitals in China, Hospital $\mathrm{F}$ is a provincial comprehensive hospital integrating medical treatment, teaching, scientific research, and preventive health care. The hospital is located in Yongwai Central Street, Donghu District of Nanchang City, covering 17.3 acres. There are many hospital departments, such as outpatient, internal medicine, surgery, emergency trauma, tumor, infection, burn, medical technology, regulation and training, scientific research, etc. There are 2900 beds and 46 clinical and medical departments in the whole hospital, which are famous for gastroenterology, neurosurgery, cardiac and vascular surgery, intensive medicine, respiratory medicine, burn, pain and emergency departments. In 2018, the outpatient volume of Hospital F reached 2.78 million.

Hospital R, formerly a church hospital founded by American Methodist Church, is the first western medicine hospital in Nanchang and the largest Grade A tertiary referral hospital under the Health Committee of Jiangxi Province. The hospital is located in Aiguo Road, Donghu District of Nanchang City. The headquarters of Hospital $\mathrm{R}$ covers an area of 7.7 acres with 2342 employees. There are many hospital departments, such as outpatient department, emergency department, inpatient department one and two, oncology radiotherapy room and dual-source computer tomography (DSCT), etc. There are 2000 beds and 57 clinical and medical departments in the whole hospital, which are famous for respiratory, digestive, cardiology, nursing specialty departments and intensive care units (ICU).

Hospital Z is a comprehensive Grade A tertiary referral hospital with unique features of tumor diagnosis and treatment, and plastic repair. Hospital $\mathrm{Z}$ is located in Beijing East Road, Qingshanhu District of Nanchang City, covering 20.3 acres. The hospital includes the outpatient department, emergency department, old and new inpatient department, laboratory department, medical technology center, radiotherapy center and internal medicine center in the hospital, with 1607 employees. There are 1650 beds and 22 clinical departments in the whole hospital, which integrate tumor prevention, diagnosis and treatment, teaching, scientific research and rehabilitation services.

Table 1 below indicates the environment of transiting 
and resting in the outpatient lobbies of three hospitals. The three hospitals have divided several areas for consultation, labor, self-services, and rest, which helps patients choose different services according to their demands and preferences. The three hospitals have posted guiding signs on the ground or the wall, assisting patients to find their ways easier. However, the research team observed that some signs did not clearly indicate directions, and some signs are severely damaged, which is difficult for patients to read and follow. The three hospitals have set up bench seats according to the number of outpatients and the structure of outpatient lobbies. However, the observation results show that the current number of bench seats still cannot adequately meet patients' demands. Taking Hospital $\mathrm{Z}$ as an example, the utilization rate of eight massage chairs is lower than expected. Besides, the problem of insufficient seats is not solved by the hospital. The lifts of three hospitals are all set to stop in single or double layers to improve the efficiency, but it also causes many patients to go back and forth often because they take the wrong lift. The escalators in Hospital $\mathrm{Z}$ effectively alleviate the massive flow of people in the outpatient lobby, but installing escalators has higher requirements on the acreage and structure of the lobby.

Table 1. Transition and rest environment in outpatient lobbies of three hospitals

\begin{tabular}{|c|c|c|c|c|c|c|c|c|}
\hline \multirow{2}{*}{ Name of hospital } & \multirow{2}{*}{ Number of areas } & \multicolumn{3}{|c|}{ Guidance signs } & \multicolumn{2}{|c|}{ Number of bench seats } & \multicolumn{2}{|c|}{ Number of elevators } \\
\hline & & Signs on the ground & Signs on the wall & Voice prompt & Ordinary chair & Massage chair & Lift & Escalator \\
\hline F Hospital & 7 & 8 & 3 & Yes & 178 & 0 & 4 & 0 \\
\hline R Hospital & 6 & 7 & 6 & Yes & 36 & 0 & 3 & 0 \\
\hline Z Hospital & 5 & 29 & 2 & No & 51 & 8 & 4 & 1 \\
\hline
\end{tabular}

Source: Observation data of the research team in July 2019

Table 2 indicates an overview of outpatient services in three hospitals. All three hospitals have set up service windows for priority groups like pregnant women, retired cadres and patients over 70 years old. When patients line up in a long queue at the ordinary service windows, the priority service windows will not help to share the registration and payment work. The three hospitals have introduced self-service equipment and encouraged patients to use self-service machines to replace manual service windows. The number of patients who prefer manual service windows is higher than those who use self-service equipment.
Meanwhile, patients using self-service equipment need manual assistance and guidance. The three hospitals have classified the medication windows to improve efficiency. However, due to a large number of patients, there are still long queues in front of the windows. There are several referrals in outpatient lobbies of three hospitals to assist patients, but their identities are different. Referrals from Hospital $\mathrm{F}$ are medical staff. Hospital $\mathrm{R}$ is attempting to introduce volunteers as guiders. A security guard from Hospital $\mathrm{Z}$ also works as a referral to assist patients in using self-service equipment.

Table 2. Overview of outpatient services in three hospitals

\begin{tabular}{|c|c|c|c|c|c|c|c|c|c|c|}
\hline \multirow{2}{*}{$\begin{array}{c}\text { Name of } \\
\text { hospital }\end{array}$} & \multicolumn{4}{|c|}{ Number of service windows } & $\begin{array}{c}\text { Number of self-service } \\
\text { equipment }\end{array}$ & \multirow{2}{*}{$\begin{array}{c}\text { Medication } \\
\text { windows }\end{array}$} & \multicolumn{4}{c|}{ Number of referrals } \\
\cline { 2 - 11 } & Normal & Priority & Refund & $\begin{array}{c}\text { Problem- } \\
\text { solving }\end{array}$ & All-in-one & Reporting & Medical staff & Volunteer & Security guard \\
\hline F Hospital & 0 & 1 & 1 & 2 & 51 & 9 & 11 & 4 & 0 & 0 \\
\hline R Hospital & 12 & 1 & 0 & 0 & 10 & 2 & 8 & 5 & 3 & 0 \\
\hline Z Hospital & 7 & 1 & 0 & 0 & 7 & 1 & 3 & 4 & 0 & 1 \\
\hline
\end{tabular}

Source: Observation data of the research team in July 2019. 


\section{Results}

\subsection{Incompetent Guiding Signs and Voice Prompt Systems}

During the interview, the research team found that elderly patients tend to seek help from the medical staff in the consultation desk and outpatient service center instead of using guide signs, mainly for the following reasons. First, most of the guiding signs in the outpatient lobby are pasted on the ground. Because the lobby is usually crowded, it is difficult for elderly patients to follow the guidance under their feet. Second, the elderly patients pointed out that the guiding signs on the ground are severely damaged, making them difficult to read and follow. Third, some elderly patients show confusion on the guiding signs because some signs cannot indicate the directions. Sometimes there can be several arrows included in one sign, and sometimes the content of the sign is difficult to read and understand. Occasionally when the elderly use the signs, they may spend lots of time going around because the signs are not clear to understand. Besides, the elderly patients reflected that the volume of prompt voice is too low to be heard. Many people have to queue again because they missed the voice messages.

\subsection{Difficulties of Transition and Rest}

According to the interview of elderly patients, they need to go between floors to access registration, medical treatment, payment, examination and medication. The elderly encountered many difficulties in transiting. Firstly, the waiting and riding time of the lifts are too long and inefficient. The elderly often rely on elevators to transit between floors, but they have to wait for a long time to get on the elevators and stay in the crowded space, sometimes they arrive on the wrong level because the lifts stop single or double levels. The elderly wasted plenty of time in transit and can feel tired physically and mentally. Secondly, there are potential risks for the elderly to use escalators. Even though some hospitals improve patients' transition efficiency by installing escalators, the elderly think that escalators move very fast. It is difficult for the elderly with visual and physical disabilities to use escalators due to potential risks of falling and dizziness ${ }^{[1]}$. In addition, elderly patients find it difficult to get a good rest while waiting for outpatient services. First, the number of bench seats is not enough; second, there is a distance between rest areas and waiting areas; third, the calling system of some waiting areas is invalid and people still need to queue up. Those reasons cause great inconvenience when they are waiting for medical treatment.

\subsection{Difficulties in Using AI Equipment}

The installation of self-service equipment brings convenience to young patients, while elderly patients feel challenging to learn to use high-technology equipment. The manual registration services and payment windows in some hospitals have been cancelled, and both registration and payment need to be carried out on WeChat public account or self-service machine. Some elderly patients said they could not use smartphones and don't have WeChat accounts, making it difficult to register and pay for themselves. Some elderly patients from rural areas told the researchers that they have difficulty operating the self-service machine because they had never seen one in rural hospitals and their education levels are limited. The elderly also reported that there is few medical staff in the self-service area to assist them and provided simple answers, which could not relieve their anxiety about using AI technology. Besides, the elderly patients also found difficult in paying fees. In many hospitals, self-service devices require patients to pay with mobile payments like WeChat, Alipay or credit cards, and elderly patients who are used to paying in cash can only queue at manual service windows. Those unnecessary transitions not only waste plenty of time but also cause the physical tiredness of elderly patients.

\subsection{Communication Barriers between Elderly Pa- tients and Medical Staff}

There is an imbalance of information between patients and doctors, which is particularly evident in elderly patients. During the interview, many elderly patients said that they have limited education level and medical knowledge, and they can feel the barriers when communicating with doctors. The elderly hope to get more information about their illness, treatment plan, medical expenses and daily precautions from doctors. However, doctors have no time to answer all the questions raised by patients due to a heavy workload. The demands of elderly patients for the explanation of illness and counseling can hardly be met ${ }^{[2,3]}$. Elderly patients also mentioned that they could scarcely understand the terms in the doctor's advice and certificate. It's difficult to find which floor they need to go for what kind of check, so they often ask medical staff repeatedly. The elderly have difficulties understanding the details of medical expenses and medical insurance and often forget the direction of medication after going home, which makes them feel worried about accessing medical services independently ${ }^{[4]}$.

DOI: https://doi.org/10.30564/jgm.v2i1.2233 


\section{Discussion}

\subsection{Optimizing the Guiding Signs and Voice Prompt Systems according to the Characteristics of Elderly Patients}

Optimizing the guiding signs and voice prompt systems is the most cost-saving way to create a suitable-for-aging environment regardless of hardware conditions. However, hospitals should consider more humanized thinking and design in the process of transformation. First of all, hospitals should take into account the visual defects of the elderly and pay attention to their blind spots. To attract the elderly's attention for the first time, hospitals should ensure the complementarity between the floor signs and the wall signs. The damaged floor signs and wall signs should be removed and repaired in time to avoid misleading patients. Secondly, hospitals should design guiding signs reasonably by considering the physiological characteristics of the elderly. The size of the signs and the characters should be taken into account the visual aspects of the elderly. Fonts of signs can be classified according to the importance, the colors can be distinguished according to the type of signs, and graphics or characters can be selected according to the practical degree ${ }^{[5]}$. Thirdly, it is necessary to combine the guiding signs with voice prompt systems to help the elderly overcome hearing or visual impairment. Elderly with hearing problems often rely on words or pictures habitually, and elderly with poor eyesight may need auxiliary voice prompts. Voice prompt can be used not only to guide the way for elderly patients but also can remind elderly patients for the call in the waiting area. It is necessary to increase the voice volume properly by considering the noisy environment.

\subsection{Designing the Areas of Transition and Rest Reasonably}

Most elderly patients reflected the difficulties when they were transiting and resting, which puts forward practical requirements for the improvement and optimization of the outpatient environment. There are three following suggestions:

First, design the elevator in the way convenient for elderly patients. Hospitals can remind the elderly patients through the guiding signs and voice prompt systems near the lifts, and can also organize volunteers near the lifts to assist elderly patients; Hospitals with escalators should adjust the escalator speed reasonably, to avoid the inconvenience caused by faster pace to the elderly. Volunteers can assist unaccompanied elderly patients with physical disorders to transit them in the process of medical treat- ment. Second, increase the number of payment counter on each floor to avoid the elderly patients going around floors. Most elderly patients said they had to go back and forth between each treatment area and the lobby to complete payment and spent a lot of time waiting for the elevator and queuing at the window. To optimize the environment, hospitals can try to add payment counters on each floor, and capable hospitals can take the lead in introducing the payment service between clinics in a pre-paid way to reduce the number of queues in the service windows. Third, plan the number and position of the bench seats. While adequately increasing the number of seats, hospitals should rationally plan the locations of seats. It is necessary to combine the rest area with the waiting area ${ }^{[6]}$. At the same time, making effective use of the voice calling system can reduce the time of standing when the elderly patients wait for consultation and reduce the anxiety of the elderly patients when they sit down and wait

\subsection{Enhancing the Ability of Elderly Patients to Use Self-service Equipment}

The introduction of self-service equipment should be a gradual process, which should not bring inconvenience to elderly patients when they access to outpatient services. On the one hand, hospitals need to consider the demands of different groups and keep an appropriate number of manual service windows to provide registration and payment services for elderly patients. On the other hand, when promoting self-service equipment, hospitals should optimize the interface and function of the equipment based on the elderly's characteristics. First, the self-service device interface should be simple, easy to understand, and the font size should be moderate, which is convenient for the elderly to identify and operate. Second, self-service equipment should combine visual and auditory effects, and assist the users' manual operation by using voice prompts to bring convenience for the elderly with visual impairment. Third, it is necessary to consider the elderly's preference for cash payment and improve the cash payment function of self-service equipment. Meanwhile, more medical staff and volunteers should be organized beside the self-service equipment to assist elderly patients in getting familiar with the use of equipment by explaining and demonstrating the functions and operation methods.

\subsection{Promoting the Medical Treatment Process to the Elderly in a Humanized Way}

Based on implementing the measures, medical staff and volunteers should try to publicize the consultation process to the elderly in a humanized way. For example, the con- 
sultation desk's medical staff can introduce registration, examination, and payment procedures to the elderly using tools such as cards and notes. This way can ensure the elderly know the distribution of the service windows and consultation areas in the hospital and eliminate the confusion and anxiety of the elderly when they find space and place. Doctors should also consider the characteristics of the elderly, such as low educational level, limited medical knowledge, decreased vision and hearing, and briefly introduce the illness, treatment plan, medical expenses and daily precautions. This can reduce the fear of elderly patients and increase their trust in medical staff. Nurses and volunteers in the outpatient service center can use easy-to-understand language to assist the elderly in completing payment, examination and medication. They can also help to explain the examination results and doctor's advice for the elderly and help the elderly understand the composition of medical expenses and medical insurance. The medication window can optimize services in a more humanized way. For example, recording the medicines' directions for elderly patients to ensure that they can understand the usage and dosage of the medication after leaving the hospital. This series of work will encourage and support elderly patients to independently access medical treatment in the future.

\section{Acknowledgments}

This paper is a part of the 'Research on the Optimization of Medical Services for the Elderly under the Healthy Jiangxi Strategy' (SH18204). This project was also sponsored by SRF for ROCS, SEM, and was supported by Postdoctoral Science Foundation in Jiangxi Province (2019KY02). In the process of the study, I want to express my appreciation to everyone who has helped me for the kindness.

Firstly, I appreciate all the members in my group for discussing, researching and translating. Some of them are still students, but they helped me a lot, like searching for documents and analyzing the data.
Secondly, my sincere appreciation also goes to the School of Humanities of Jiangxi University of Finance and Economics colleagues for their valuable advice.

Lastly, I shall extend my thanks to the anonymous reviewers for your hard-working and thorough attitude towards the paper.

\section{References}

[1] Simón M, Giraldo L, Sommer J, et al. Fall Risk Assessment Through a Self-Service Terminal in the Outpatient Setting[J]. Stud Health Technol Inform, 2019, 8(264): 1352-1355.

\section{DOI: 10.3233/SHTI190447}

[2] Chunyan Xie, Wendi Cheng, Changying Wang, et al. Dilemma and Countermeasures of Doctor-patient Relationship Based on the Logic of Imbalance between Supply and Demand in Health Care Services[J]. Chinese Health Resources, 2017, 20(3): 263-267.

\section{DOI: 10.13688/j.cnki.chr.2017.16752}

[3] Danqian Dai. Problems and Measures of the Doctor-patient Relationship [J]. Chinese Health Service Management, 2008, (4): 237-238.

[4] Tseng, Ming-Hseng, Wu, Hui-Ching. A Cloud Medication Safety Support System Using QR Code and Web Services for Elderly Outpatients[J]. Technology and Health Care, 2014, 1(22): 99-113.

\section{DOI: 10.3233/THC-140778}

[5] Qing Zhang, Yongguan Cui, Maohui Huang. Basic Thinking of Designing a Hospital Guidance Identification System[J]. Chinese Journal of Hospital Administration, 2004, 20(10): 629-630.

\section{DOI: 10.3760/j.issn:1000-6672.2004.10.023}

[6] Ahmadi Teymourlouy, A, Seyyedin, H, Fadaye-vatan, R, et al. Assessing the Suitability of the Design, Safety and Physical Environment of Hospitals for the Elderly: A Case Study in Iran[J]. Iranian Journal of Health, Safety and Environment, 2019, 6(1): 12131220. 